\title{
A Novel Model for Contractor Selection Decision
}

\author{
Hsien-Kuan Chang, Wen-Der Yu ${ }^{\mathrm{a},{ }^{*}}$, Yuan-Yu Hsu ${ }^{\mathrm{b}}$, Shao-Tsai Cheng ${ }^{\mathrm{b}}$, Kwo-Wuu Wang ${ }^{\mathrm{c}}$ \\ ${ }^{a}$ Chaoyang University of Technology, No. 168, Jifeng E. Rd., Wufeng Dist., Taichung, 41349 Taiwan \\ ${ }^{b}$ Chung Hua University, No. 707, Sec.2, WuFu Rd., Hsinchu, 30012 Taiwan, Taiwan \\ ${ }^{b}$ National Defense University, No. 1000, XingFeng Rd., Bade Dist., Taoyuan City 334, Taiwan
}

\begin{abstract}
The prevailing practice on the owner's decision of contractor selection method for a procurement project has been determined by the top manager of the procurement agency via her/his comprehensive consideration of all affecting factors. Although previous researchers have proposed some analytic models to assist the decision of contractor selection method, the procurement personnel usually tend to adopt Lowest Tender (LT) for contractor selection to avoid violation of the regulation for 'Abuse of Public Power for Private Profit' without a no clear evidence to support the adoption heterogeneous procurement method (HPM), e.g., Best Value (BV) or Heterogeneous Lowest Tender (HLT). Moreover, all previous contractor selection analytic models employ the posttendering data, it is impossible to be applied for pre-tendering decision making. To improve this drawback, the current paper proposes a Pre-tendering Graphic Analytic Model (Pre-GAM) that builds the required graphical analytic model for decision of contractor selection method using a special standardization method for historical bidding data. A real-world building construction project is adopted to demonstrate practical application and to show that the proposed Pre-GAM is able to improve the problem of post-tendering analysis and determine the correct contractor selection method.
\end{abstract}

(C) 2019 The Authors. Published by Budapest University of Technology and Economics \& Diamond Congress Ltd.

Peer-review under responsibility of the scientific committee of the Creative Construction Conference 2019.

Keywords: Heterogeneous procurement, Graphical analysis model, Standardization, Bidding data.

\section{Introduction}

Construction projects are usually delivered by the contractor on a contracting basis. The bidders compete for the project and the winner is awarded with the contract via a procurement process. It is therefore very important to select the most appropriate contractor for a construction project in order to achieve both successful and quality project delivery and also cost-effectiveness. Due to the regulation of Government Procurement Agreement (GPA) under World Trade Organization (WTO) [1], the public construction agencies of the Government of GPA signatory countries are required to conduct project procurement process following the international standards. In compliance with the GPA requirements, the Taiwan Government enacted the Government Procurement Law (GPL) to regularize the procurement process. There are two most commonly adopted project procurement methods defined in GPL [2]: (1) the Lowest Tender (LT) - the contractor with lowest bid wins the contract and it is more appropriate for homogeneous procurement projects; (2) the Best Value (BV) - the contractor with highest value (conceived by the procurement agency) wins the contract and it is more appropriate for heterogeneous procurement projects. Another new method for project procurement was proposed in the past decade, namely the Heterogeneous Lowest Tender (HLT), which was 
Hsien-Kuan Chang, Wen-Der Yu, Yuan-Yu Hsu, Shao-Tsai Cheng, and Kwo-Wuu Wang / Proceedings of the Creative Construction Conference (2019) 026

https://doi.org/10.3311/CCC2019-026

designed for procurement projects whose heterogeneity is intermediate, between heterogeneous and homogeneous [3]. According to the statistics of the Public Construction Commission (PCC) of Taiwan Government and results of previous researchers, it shows that BV and HLT outperformed the LT in project delivery performance [4], while the HLT outperforms BV in cost-effectiveness if it is adopted in the appropriate project type [5]. However, a difficult decision must be made by the procurement agency that whether the project is heterogeneous enough for either BV or HLT, since the public procurement personnel usually tend to adopt LT for procurement in order not to violate the law [6]. Under such circumstance, the public project owners will not be able to benefit from heterogeneous procurement methods. There have been several models proposed by the previous researchers to evaluate the heterogeneity of the procurement project, including P/PAM [6], P/PAM-GAM [7] and GAM-PEP [8]. One key information required for all the previous models is the Market Price Elasticity of Performance (annotated as $\mathrm{E}_{\mathrm{m}}$ ), or Market PEP [9]. The value of $\mathrm{E}_{\mathrm{m}}$ needs to be determined by plotting the Performance/Price diagram (Q-P Diagram) with the tendering information (i.e., 'evaluated performance score' vs. 'bidding price') of the potential bidders in the heterogeneous procurement market [6]. However, the previous models required the tendering information after the potential bidders submitted their bids. This is impractical for the application of real-world projects, since the project procurement method needs to be determined before the Request for Proposal (RFP) or Request for Quotation (RFQ) is announced. Such models can be categorized as 'post-tendering evaluation models' that can be used to evaluate whether the decision on procurement method selection is appropriate or not, they are however not useful for the procurement personnel of the agency to make appropriate decision on which procurement method should be selected. In order to support the procurement method selection decision, this paper proposes a 'pre-tendering graphic analysis model', namely Pre-GAM, for determination of the most appropriate project procurement method for a construction project.

The rest of the paper is presented as follows: the important relevant works are reviewed in Section 2; then the research methodology is explained in Section 3; in Section 4, the proposed Pre-GAM is described in detailed; a real-world example of residential building construction project is selected for case study in Section 5; finally, conclusions and recommendations are addressed in Section 6.

\section{Relevant Works}

As addressed previously, the critical point to select the most appropriate procurement method is to determine the 'heterogeneity' of the project. The 'heterogeneity' of a project is actually determined by the capability of the contractors available in the market, who are able to deliver the project results with different performances, e.g., time, cost, image, aesthetics/appearance, operation and maintenance, managerial safety and environmental aspects, and many other factors $[10,11,12]$. If the procurement market is relatively highly heterogeneous, the heterogeneous procurement method such as BV and HLT should be adopted; otherwise, the homogeneous procurement method (e.g., LT) should be adopted. There have been primarily two approaches to evaluate the heterogeneity of the contractors in delivering heterogeneous project performances: (1) the subjective approach - the overall project heterogeneity is determined solely by the manager of the procurement agency, and the heterogeneous performance of the individual tender is evaluated by the Procurement Evaluation Committee (PEC) [13,14]; (2) the objective approach-the overall project heterogeneity is determined solely by the market or historical performance data of the contractors [15,6,9]. Although the subjective approach is more easily implemented, it is less reliable and doesn't guarantee the optimal result. The objective approach is more reliable and may lead to optimal decision if sufficient historical tendering data are available.

$\mathrm{Yu}$ and Wang [9] proposed a quantitative indicator, namely 'Price Elasticity of Performance (PEP)' or 'E', to measure the heterogeneity of the procurement market. The PEP of an individual tender was defined by Yu and Wang [12] as follows: 
Hsien-Kuan Chang, Wen-Der Yu, Yuan-Yu Hsu, Shao-Tsai Cheng, and Kwo-Wuu Wang / Proceedings of the Creative Construction Conference (2019) 026

https://doi.org/10.3311/CCC2019-026

$$
P E P=\frac{\Delta Q / Q}{\Delta P / P}=\frac{\Delta Q}{\Delta P} \times \frac{P}{Q}=\frac{1}{m} \times \frac{P}{Q}
$$

Where, $E$ is the measurement of PEP, $Q$ is the measure of work performance, $\Delta Q / Q$ is the percentage of change in work performance, $P$ is the price, $\triangle P / P$ is the percentage of change in the price, and $m$ is the slope of the tangent at point $(Q, P)$ in the Performance/Price diagram (Q-P Diagram).

Considering all contractors bidding for the same project as a 'market', the collective characteristics of these contractors in the market, i.e., 'market PEP' or $\mathrm{E}_{\mathrm{m}}$, and has been defined in by Wang and $\mathrm{Yu}$ [6].

Several project heterogeneity evaluation methods were proposed by Yu et al. [16], Wang and Yu [7] and Wang et al. [8] based on the indicator of market price elasticity of performance $\left(\mathrm{E}_{\mathrm{m}}\right)$ [9]. Although the $\mathrm{E}_{\mathrm{m}}$ provides a handy method for measuring the heterogeneity of the procurement market, it needs the tendering information to plot the required QP Diagram. While the tendering information is available only after the tenders are submitted by the bidders, it is not useful for the decision making when the project procurement method needs to be determined. Such a limitation is common for all PEP-based evaluation methods, e.g., P/PAM [6], P/PAM-GAM [7] and GAM-PEP [8].

\section{Research Methodology}

The research aims at resolving the post-tendering problem of the previously proposed PEP-based project heterogeneity evaluation methods and proposing a pre-tendering method for measuring the market price elasticity of performance $\left(\mathrm{E}_{\mathrm{m}}\right)$ to evaluate the heterogeneity of a specific procurement project in order to select the most appropriate project procurement method (i.e., LT, BV or HLT).

In order to attain the defined research objectives, the Q-P Diagram of a procurement market has to be established with the historical tendering data, including the 'performance score (PS) vs. bidding price (BP)' datasets, which should be collected from former procurement projects of a specific owner. After data collection, the following data analysis process is conducted:

- Standardization of historical tendering data - since the historical tendering data are collected from different project, the first step is to standardize these data so that they can be utilized in constructing the same Q-P Diagram. There are two types of tendering information, the 'performance score (PS) / bidding price (PB)' of a specific bidder in the historical project. They are processed separately.

- Construction of Q-P Diagram - the Q-P Diagram of P/PAM for measuring the heterogeneity of a procurement market is constructed with the standardized historical tendering data.

- Development of procurement method selection criteria - the criteria for selecting the most appropriate project procurement method are developed according to the heterogeneity of procurement market, the project requirement of performance (PRP), and the characteristics of bidding contractors.

- Compilation of the Pre-tendering Graphic Analysis Model (Pre-GAM model) for procurement method selectionfinally, the Q-P Diagram constructed based on historical tendering data, the procurement method selection criteria, and the determination of project procurement method are compiled to verify the feasibility of the proposed Pre-GAM model for the demonstrated case project.

\section{Proposed Pre-GAM model for Residential Building Projects}

In this section, the proposed Pre-GAM model is developed specifically to support the procurement method decision for a specific procurement agency. 
Hsien-Kuan Chang, Wen-Der Yu, Yuan-Yu Hsu, Shao-Tsai Cheng, and Kwo-Wuu Wang / Proceedings of the Creative Construction Conference (2019) 026

https://doi.org/10.3311/CCC2019-026

\subsection{Collection and standardization of historical tendering data}

In the first step, the tendering data need to be collected from historical projects. The basic information of the five historical building projects are shown in Table 1.

Table 1. Basic information of the five historical building procurement projects.

\begin{tabular}{llllll}
\hline Project ID & A & B & C & D & E \\
\hline Building type & 5 -floor elev. apartment & 5-floor elev. apartment & 5-floor apartment & 5 -floor elev. apartment & 5-floor townhouse \\
Site area $\left(\mathrm{m}^{2}\right)$ & 364 & 496 & 584 & 167 & 330 \\
Floor area $\left(\mathrm{m}^{2}\right)$ & 1655 & 1690 & 1963 & 727 & 958 \\
Budget (USD) & 733,333 & 3100 & 3700 & 1350 & 2100 \\
PRP (score \%) & 70 & 70 & 70 & 70 & 70 \\
\hline
\end{tabular}

The tendering data associated with the five historical building projects are also collected and shown in Table 2.

Table 2. Tendering data of the five historical building procurement projects.

\begin{tabular}{cccccccccccc}
\hline Project ID & \multicolumn{2}{c}{ A } & \multicolumn{2}{c}{ B } & \multicolumn{3}{c}{ C } & & D & & E \\
\hline Contractor & PS & BP & PS & BP & PS & BP & PS & BP & PS & BP \\
\hline I & 71.27 & 537,000 & 71.98 & 765,667 & 72.27 & 925,333 & 72.48 & 341,333 & - & - \\
II & - & - & - & - & 70.01 & 915,000 & 70 & 343,000 & 70 & 522,000 \\
III & 75.38 & 630,667 & 74.89 & 892,333 & 75.99 & $1,073,000$ & 75.92 & 388,667 & 75.82 & 603,000 \\
IV & - & - & 74.75 & 801,000 & - & - & - & - & 75.24 & 539,000 \\
V & 81.75 & 668,000 & - & - & 82.14 & $1,116,333$ & 82.12 & 410,000 & - & - \\
VI & 84.72 & 685,000 & 83.83 & 984,000 & 85.32 & $1,160,667$ & - & - & 84.55 & 668,000 \\
VII & - & - & 80.98 & 885,333 & - & - & 82.06 & 390,667 & 81.75 & 606,333 \\
VIII & 75.52 & 552,333 & - & - & 76.66 & 930,333 & 76.03 & 343,333 & 75.78 & 536,000 \\
IX & 85.02 & 634,667 & - & - & 83.92 & $1,082,667$ & 84.27 & 393,667 & - & - \\
\hline
\end{tabular}

Since the scopes of the five historical projects of Table 2 are different, the tendering data need to be pre-processed before they are used to construct the Q-P Diagram for the building construction procurement market of the project owner. A standardization method for PS/BP data is proposed comprising the following steps:

- Collecting tendering data from historical projects - collect the PS/BP data (e.g., the tendering data of Table 2) for the same procurement agency (or project owner);

- Categorizing the project types - group the tendering data according to project types to categorize different procurement markets;

- Standardizing the performance score (PS) and bidding price (BP) data using the Eq. (4) and Eq. (5), respectively.

Eq. (4) is used for PS standardization:

$$
\mathrm{Q}_{i j}=\frac{\text { Score }_{i j}}{\text { MaxScore }_{j}} \times 100 \%
$$

Where, Score $_{i j}$ is the original performance score (PS) of the $i^{\text {th }}$ contractor/bidder for the $j^{\text {th }}$ project; MaxScore $e_{j}$ is the maximum score (usually use $100 \%$ as the maximum score) for the $j^{\text {th }}$ project; $Q_{i j}$ is the standardized PS of the $i^{\text {th }}$ contractor/bidder for the $j^{\text {th }}$ project.

Similarly, Eq. (5) is used for BP standardization: 
Hsien-Kuan Chang, Wen-Der Yu, Yuan-Yu Hsu, Shao-Tsai Cheng, and Kwo-Wuu Wang / Proceedings of the Creative Construction Conference (2019) 026

https://doi.org/10.3311/CCC2019-026

$$
\mathrm{P}_{i j}=\frac{\text { Price }_{i j}}{\text { Budget }_{j}} \times 100 \%
$$

Where, Price $_{i j}$ is the original bidding price (BP) of the $i^{\text {th }}$ contractor/bidder for the $j^{\text {th }}$ project; $B$ Budget is $_{j}$ the budget for the $j^{\text {th }}$ project defined by the owner; $P_{i j}$ is the standardized BP of the $i^{\text {th }}$ contractor/bidder for the $j^{\text {th }}$ project.

\subsection{Construction of standardized $Q-P$ Diagram}

After standardizing the historical tendering data, the standardized $\mathrm{PS}\left(\mathrm{Q}_{\mathrm{ij}}\right)$ and $\mathrm{BP}\left(\mathrm{P}_{\mathrm{ij}}\right)$ for a specific contractor/bidder is obtained by averaging the standardized PS/BP data of all historical projects for this contractor/bidder. The standardized PS/BP data points of all contractors/bidders in the market are plotted as the ' $\mathrm{x}$ ' points in the standardized historical Q-P Diagram, shown in Fig. 1.

There are some important features of the constructed standardized historical Q-P Diagram in Fig. 1: (1) the upperbound and lower-bound both for the PS $(\mathrm{Q})$ and $\mathrm{BP}(\mathrm{P})$ are outlined to highlight the boundary of the feasible tendering zone, since the performance score are required to be higher than the lower-bound and bidding price is required to be lower than the budget; (2) using the method proposed by Yu and Wang [9], the highest-quality curve (HQ) and the lowest-quality curve (LQ) are generated with general quadratic equations as depicted in the figure; (3) the market PEP $\left(E_{m}\right)$ can be estimated with the slope of the straight line connecting the two intersection points of HQ and LQ. As the estimated $\mathrm{E}_{\mathrm{m}}=0.8313$, thus it is a relatively homogeneous procurement market.

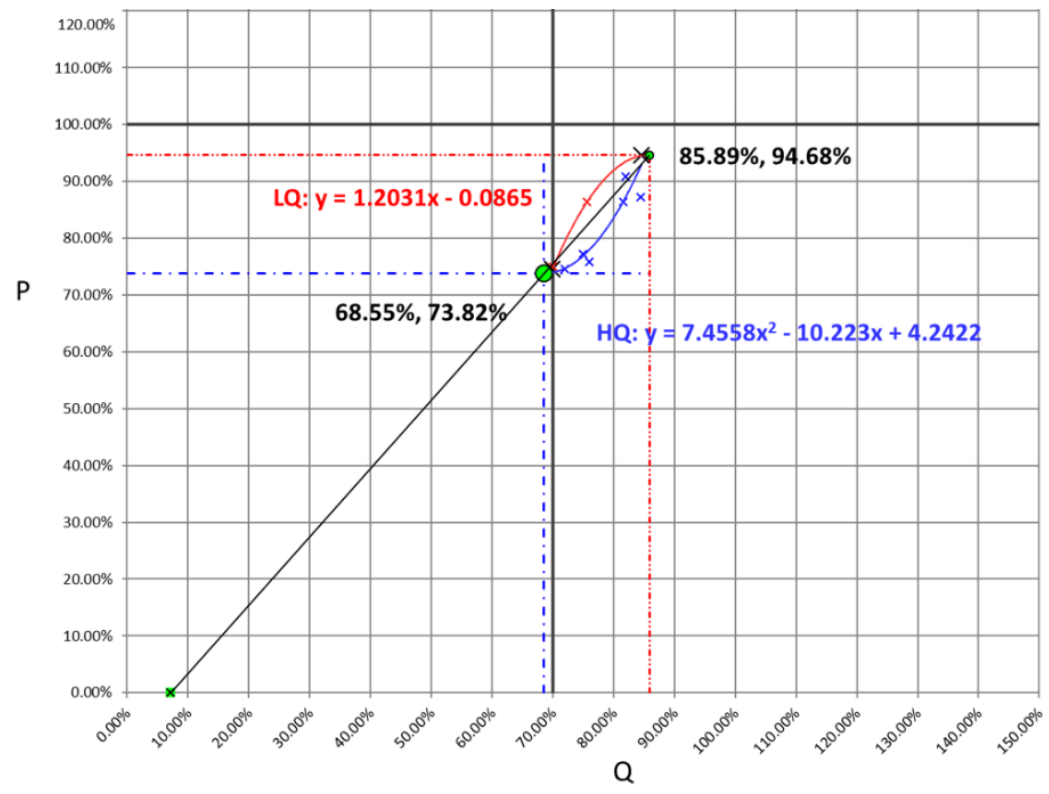

Fig. 1. Standardized Q-P Diagram of historical building projects.

\subsection{Development of procurement method selection criteria}

Refer to Fig. 1, where there are three critical parameters that will affect the result of procurement: (1) Lower-bound of performance $\left(\mathrm{Q}_{\min }\right)$ — only the tender with the evaluated performance $\mathrm{Q}_{\min }(\mathrm{e} . \mathrm{g}$., 70\%) can be awarded; (2) Minimum of the submitted bidding prices $\left(\mathrm{P}_{\min }\right)$ — only the tender with bidding price (at least the $\mathrm{P}_{\min }$ of the submitted bidding prices) less than budget can be awarded; (3) Market PEP ( $\left.E_{m}\right)$ - as the higher the value of $E_{m}$, the higher the heterogeneity the market is and thus the heterogeneous procurement method should be adopted. As a result, the 
Hsien-Kuan Chang, Wen-Der Yu, Yuan-Yu Hsu, Shao-Tsai Cheng, and Kwo-Wuu Wang / Proceedings of the Creative Construction Conference (2019) 026

https://doi.org/10.3311/CCC2019-026

procurement method selection criteria are summarized in Table 3. Referring to the values of the above-mentioned parameters, the procurement method selection criteria can be planned as follows:

- If the $\mathrm{Q}_{\min }$ is $\geqq$ Lower-bound and $\mathrm{E}_{\mathrm{m}}$ is $<1.0$, it can be classified as a relatively lowly heterogeneous market. However, $\mathrm{Q}_{\min }$ is < Lower-bound, it is recommended to adopt HLT to prevent the disqualified contractors from awarding, no matter the submitted prices of the bidders.

- If the $\mathrm{Q}_{\min }$ is $\geqq$ Lower-bound and $\mathrm{E}_{\mathrm{m}}$ is $\geqq 1.0$, it can be classified as a relatively highly heterogeneous market. However, $\mathrm{Q}_{\min }$ is < Lower-bound, it is recommended to adopt BV if there exists a bidder with $\mathrm{P}_{\min }<100 \%$. However, if the $\mathrm{P}_{\min }$ of all bidders are $\geqq 100 \%$, it implies that the procurement may be over-budget, thus HLT should be adopted to lower the cost.

- If the $\mathrm{Q}_{\min }$ is $\geqq$ Lower-bound and $\mathrm{E}_{\mathrm{m}}$ is $<1.0$, it can be classified as a highly homogeneous market and all contractors are qualified. In such a case, it is recommended to adopt LT in order to lower the cost.

- If the $\mathrm{Q}_{\min }$ is $\geqq$ Lower-bound and $\mathrm{E}_{\mathrm{m}}$ is $\geqq 1.0$, it can be classified as a highly heterogeneous market and all contractors are qualified. In such a case, BV should be adopted if the $\mathrm{P}_{\min }<100 \%$. However, if the $\mathrm{P}_{\min } \geqq 100 \%$ it is recommended to adopt LT to lower the cost.

Table 3. Procurement method selection criteria.

\begin{tabular}{lllll}
\hline$Q_{\text {min }}$ & $<$ Lower-bound (70\%) & \multicolumn{2}{l}{$\geqq$ Lower-bound (70\%) } \\
\hline$E_{m}$ & $E_{m}<1.0$ & $E_{m} \geqq 1.0$ & $E_{m}<1.0$ & $E_{m} \geqq 1.0$ \\
\hline$P_{\min }<100 \%$ & HLT & BV & LT & BV \\
$P_{\text {min }} \geqq 100 \%$ & HLT & HLT & LT & LT \\
\hline
\end{tabular}

\section{Application Demonstration}

In this section, a new building construction procurement project is tested with the proposed Pre-GAM for the procurement method selection of a specific building project owner to demonstrate its applicability. The testing case project is a 5-floor apartment with elevator. Project site area is $167 \mathrm{~m}^{2}$ and the floor area is $730 \mathrm{~m}^{2}$, with project budget of USD\$ 483,330 .

At first, the required parameters are calculated according to the historical tendering data of Table 2 and the procurement criteria of the case building construction project to determine the boundaries of the Q-P Diagram. The resulted Q-P Diagram is shown in Fig. 2

From Fig. 2, it can be noted that the required parameters from the historical tendering data are: $\mathrm{Q}_{\min }=53.19 \%, \mathrm{Q}_{\max }=$ $87.52 \%, \mathrm{P}_{\min }=65.89 \%, \mathrm{P}_{\max }=94.22 \%$; and the equations for the two groups of bidders are HQ: $\mathrm{y}=2.2957 \mathrm{x}^{2}-$ $2.4422 x+1.3084$ and LQ: $y=-6.1709 x^{2}+10.8012 x-3.7843$, respectively. Thus, the estimated $E_{m}=1.2118>1.0$ and it is a moderately heterogeneous procurement market. The feasible procurement zone is shown as the squired in the central area of Fig. 2. There are two parts of the squire area: the green zone in the right-hand side is feasible for HLT and BV as the $\mathrm{Q}_{\min }$ is $\geqq$ Lower-bound (70\% in this case), while the pink zone in the left-hand side is feasible for LT as the $\mathrm{Q}_{\text {min }}$ is < Lower-bound.

After determining the feasible procurement zone, a procurement evaluation board (PEB) is organized consisting of five experienced domain experts ( 2 site engineers of construction firm, 1 architect, 1 procurement staff of a government agency, and 1 scholar specialized in procurement). Totally seven contractors (B1 B7) have submitted their bids to this project. The bidding documents include the bidding price and a proposal of construction plan with previous performance information for the performance evaluation by the PEB. The tendering data of the seven bidders are shown in Table 4, where the average historical performance scores (PS) of the seven bidders are shown in Row 9, 
Hsien-Kuan Chang, Wen-Der Yu, Yuan-Yu Hsu, Shao-Tsai Cheng, and Kwo-Wuu Wang / Proceedings of the Creative Construction Conference (2019) 026

https://doi.org/10.3311/CCC2019-026

while the standardized bidding prices (BP) are shown in Row 11. The standardized PS and BP are then plotted in Fig. 2 to obtain the distribution of tendering data for the bidders in the Q-P Diagram (see the dots of Fig. 2).

From Fig. 2, it is recognized that B2 with tendering information of $(71.6 \%, 82.76 \%)$ will be awarded if LT method is adopted for procurement, while B7 with tendering information of $(84.0 \%, 92.41 \%)$ will be awarded if BV method is adopted. The BV method is recommended since all contractors offer the performance $>\mathrm{Q}_{\min }, \mathrm{E}_{\mathrm{m}} \geqq 1.0$, and $\mathrm{P}_{\min }=$ USD $\$ 400<$ budget (USD $\$ 483,000$ ). As a result, B7 should be awarded using BV method.

Table 4. Tendering data of the 7 bidders for the case building construction project.

\begin{tabular}{|c|c|c|c|c|c|c|c|}
\hline \multirow{2}{*}{ PEC } & \multicolumn{7}{|c|}{ Historical performance records of bidders } \\
\hline & $\mathrm{B}_{1}$ & $\mathrm{~B}_{2}$ & $\mathrm{~B}_{3}$ & $\mathrm{~B}_{4}$ & $\mathrm{~B}_{5}$ & $\mathrm{~B}_{6}$ & $\mathrm{~B}_{7}$ \\
\hline A & 72 & 72 & 76 & 79 & 80 & 75 & 83 \\
\hline $\mathrm{B}$ & 75 & 69 & 75 & 80 & 85 & 76 & 85 \\
\hline $\mathrm{C}$ & 73 & 71 & 74 & 76 & 83 & 74 & 88 \\
\hline $\mathrm{D}$ & 71 & 74 & 73 & 80 & 82 & 77 & 82 \\
\hline $\mathrm{E}$ & 73 & 72 & 77 & 85 & 86 & 75 & 82 \\
\hline Sum & 364 & 358 & 375 & 400 & 416 & 377 & 420 \\
\hline Standardized PS & 72.8 & 71.6 & 75.0 & 80.0 & 83.2 & 75.4 & 84.0 \\
\hline BP (1000 USD) & 410 & 400 & 400 & 429 & 447 & 427 & 447 \\
\hline Standardized BP & $84.83 \%$ & $82.76 \%$ & $82.76 \%$ & $88.83 \%$ & $92.41 \%$ & $88.28 \%$ & $92.41 \%$ \\
\hline
\end{tabular}

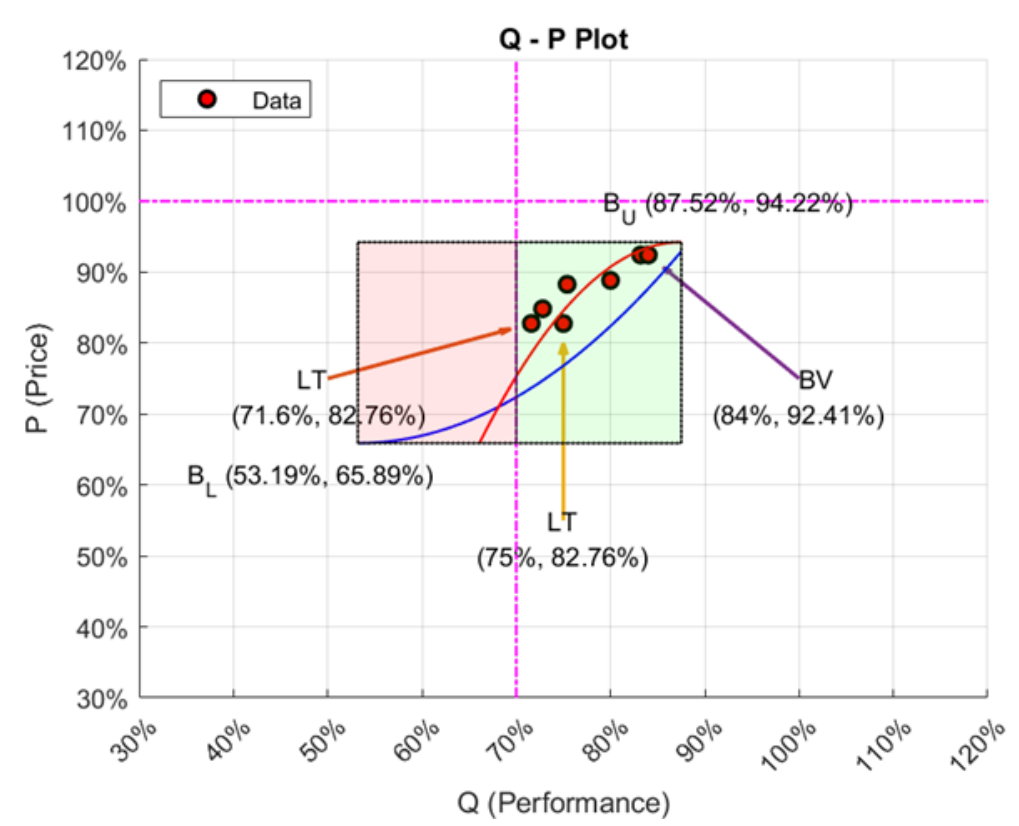

Fig. 2. Distribution of tendering data for bidders in the Q-P Diagram.

\section{Conclusion and Recommendation}

This paper proposes a Pre-tendering Graphic Analytic Model (Pre-GAM) to resolve the traditional difficult decision facing the procurement personnel in selecting the most appropriate one among the prevailing procurement methods, e.g., Lowest Tender (LT), Best Value (BV) and Heterogeneous Lowest Tender (HLT). The proposed Pre-GAM builds the required graphical analytic model for the decision of contractor selection method using a special standardization method for historical bidding data. It breaks the barrier of 'post-tendering decision-making' limitation encountered by the similar previous models including P/PAM [6,9], P/PAM-GAM [7], and GAM-PEP [8]. With such an improvement, 
Hsien-Kuan Chang, Wen-Der Yu, Yuan-Yu Hsu, Shao-Tsai Cheng, and Kwo-Wuu Wang / Proceedings of the

Creative Construction Conference (2019) 026

https://doi.org/10.3311/CCC2019-026

the project owners will be able to enjoy the benefits of heterogeneous procurement method (e.g., BV and HLT) as the decisions of the procurement personnel are logically and systematically supported.

A real-world building construction project is adopted to demonstrate the practical application and to show that the proposed Pre-GAM is able to improve the problem of post-tendering analysis and determine the most appropriate contractor selection method. The decision of procurement method selection becomes very straightforward by plotting the tendering data of the Q-P Diagram developed by Pre-GAM. The project owner can also use Pre-GAM to evaluate their decision on procurement method selection after tendering. With such a mechanism, the procurement quality will be improved in the long run, and the benefits of the owner will be better secured.

In this paper, the Pre-GAM for a building project of a specific owner is developed. Future research is recommended to conduct similar model building procedure for different government or private owners on different types of construction procurement projects to achieve better owner benefits.

\section{Acknowledgements}

This research project was funded by the Ministry of Science and Technology, Taiwan, under project No. MOST 1072221-E-324-007. Sincere appreciations are given to the sponsor by the authors.

\section{References}

[1] WTO. Agreement on Public Procurement. Government Procurement Agreement. World Trade Organization. Available online: https://www.wto.org/english/docs_e/legal_e/gpr-94_e.pdf (accessed on 1 February 2019).

[2] PCC. Government Procurement Law, Public Construction Commission. Available online: http://lawweb.pcc.gov.tw/EngLawContent.aspx ?Type=E\&id=49\&KeyWord=Government+Procurement+Act (accessed on 1 February 2019).

[3] Public Construction Commission. "Submission Notes for HLT Procurement.” PCC Official Letter, 2006.5.23 NOG: 09500191630, Public Construction Commission, 2006.

[4] T. Y. Su, "The Research of the Lowest Bid Mechanism of Heterogeneous Procurement in Public Sectors-A Case Study of the Water Resources Agency." Ministry of Economic Affairs in Taiwan. Master Thesis, Executive Master Program of Public Affairs, Tung Hai University, 2010. (in Chinese) http://dx.doi.org/10.1016/j.ijindorg.2012.02.002

[5] H. J. Chen, "The Implementation Analysis of Awarding Principles in Government Procurement Act." Master Thesis, Department of Civil Engineering, National Taiwan University, 2008. (in Chinese) https://www.mdpi.com/2071-1050/10/10/3583/htm

[6] K. W. Wang and W. D. Yu, "Model for Analysis of Heterogeneity in Product Acquisition Procurement." Journal of the Chinese Institute of Engineers, Chinese Institute of Engineers, 34 (2011) 877-887. https://doi.org/10.1080/02533839.2011.591917

[7] K. W. Wang, and W. D. Yu, “A Graphical Model for Analysis of Heterogeneous Procurement.” Journal of Technology, 29 (2014) 1-15. (in Chinese)

[8] K.W. Wang, Y.Y. Hsu, W. D. Yu, and S. T. Cheng, "Determination of Project Procurement Method with a Graphical Analytic Model." Sustainability, 10 (2018), https://doi:10.3390/su10103583.

[9] W. D. Yu, and, K. W. Wang, "Best Value or Lowest Bid? A Quantitative Elasticity Perspective." Journal of Construction Engineering and Management, ASCE, 138 (2011) 128-134.

[10] D. D. Gransberg and M. A. Ellicott, "Best-value contracting criteria.” Cost Engineering, 39 (1997) 31-34.

[11] M. Abdelrahman, T. Zayed, and A. Elyamany, "Best-Value Model Based on Project Specific Characteristics." Journal of Construction Engineering \& Management, ASCE, 134 (2008) 179-188. https://doi.org/10.1061/(ASCE)0733-9364(2008)134:3(179)

[12] M. Feldman, "Best Value in Publicly Funded Projects: Contractor Selection in Two County GOB Projects." Research Report, Center for Labor Research \& Studies (CLR\&S), Florida International University, FL, USA, 2006.

[13] B. T. Gale, and D. J. Swire, “Customer Value Accounting for Value-Based,” Journal of Professional Pricing, 15 (2006) $30-33$.

[14] W. C. Wang, W. D. Yu, I. T. Yang, and C. C. Lin, “Applying AHP to support the best-value contractor selection- lessons learned from two case studies in Taiwan," Journal of Civil Engineering and Management, 19 (2012) 24-36. http://dx.doi.org/10.3846/13923730.2012.734851

[15] B. L. Oo, D. S. Drew, and H. P. Lo, "Modeling the heterogeneity in contractors' mark-up behavior." Journal of Construction Engineering and Management. 136 (2010) 720-729. https://doi.org/10.1061/(ASCE)CO.1943-7862.0000186

[16] W. D. Yu, K. W. Wang, and M. T. Wang, "Pricing Strategy for Best Value Tender." Journal of Construction Engineering and Management. ASCE, 139 (2013) 675-684. https://doi.org/10.1061/(ASCE)CO.1943-7862.0000635 